\title{
La violence rurale dans la France du XIXe siècle et son dépérissement : l'evolution de l'interprétation politique
}

\section{Alain Corbin}

\section{OpenEdition \\ Journals}

Édition électronique

URL : http://journals.openedition.org/conflits/298

DOI : $10.4000 /$ conflits.298

ISSN : $1777-5345$

Éditeur :

CCLS - Centre d'études sur les conflits lilberté et sécurité, L'Harmattan

Édition imprimée

Date de publication : 15 mai 1993

ISSN : 1157-996X

Référence électronique

Alain Corbin, « La violence rurale dans la France du XIXe siècle et son dépérissement : I'evolution de

l'interprétation politique », Cultures \& Conflits [En ligne], 09-10 | printemps-été 1993, mis en ligne le 13 mars 2006, consulté le 30 mars 2021. URL : http://journals.openedition.org/conflits/298 ; DOI :

https://doi.org/10.4000/conflits.298

Ce document a été généré automatiquement le 30 mars 2021.

Creative Commons License 


\title{
La violence rurale dans la France du XIXe siècle et son dépérissement : l'evolution de l'interprétation politique
}

\author{
Alain Corbin
}

1 De la chute du Premier Empire au milieu des années 1850, les campagnes françaises sont traversées de vagues de violence collective. Cette agitation sporadique retombe par la suite. Entre la brève crise de 1868 et le milieu du XXe siècle, les communautés rurales vivent dans un calme jusqu'alors inconnu. Les historiens, fidèles en cela à la vision des élites de ce temps hanté par l'éventuelle renaissance de la jacquerie, ont le plus souvent, interprété la turbulence du premier XIXe siècle comme un archaïsme. Yves-Marie Bercé ${ }^{1}$ a naguère montré, de manière convaincante, comment les formes de cette violence s'inscrivaient dans la tradition des Croquants et des Va-nu-pieds. Dans une thèse récente, l'historien canadien, Denis Beliveau ${ }^{2}$, situe les quelques cinq cents troubles frumentaires de la monarchie censitaire dans le droit fil de ces "fureurs paysannes" qui ont suscité la querelle que l'on sait, entre Roland Mousnier et Boris Porchnev. Pour d'autres historiens, les mécanismes de la violence collective qui agite les campagnes prolongent ceux de la Grande Peur ou, plus largement, les gestes de la révolution paysanne de 1789, l'une et l'autre si bien analysées par Georges Lefebvre', quelque peu tributaire, à son corps défendant, de la psychologie des foules élaborée au XIXe siècle. Les attaques et les pillages de châteaux qui se sont déroulés en 1830 dans la Corrèze et dans la Dordogne ${ }^{4}$ ou dans la Basse-Marche en 1848, et ce ne sont que des exemples, confortent cette thèse de la simple réitération. La vague d'anticléricalisme qui suit la révolution de Juillet et l'incessante référence à un éventuel rétablissement de la dîme, voire du régime seigneurial, jusqu'au triomphe de la IIIe République ${ }^{5}$, contribuent à étayer cette interprétation. Considérons plus précisément les découvertes qui ont conforté cette lecture de la violence paysanne. Depuis le lancement de l'enquête collective dirigée par Ernest Labrousse à l'aube des années 1950 et consacrée à la grande crise du milieu du XIXe siècle, les troubles frumentaires ont été 
finement analysés; et cela, avant même que Denis Beliveau ${ }^{6}$ entreprenne son travail. Maurice Levy-Leboyer, Rémy Gossez, Philippe Vigier, Yvon Bionnier, sans oublier les historiens anglo-saxons déjà cités, ont mis en évidence le poids de la conjoncture, la rationalité économique et géographique de cette agitation, dont ils ont, en outre, analysé précisément les procédures ${ }^{7}$. Edward-P. Thompson, à propos des foules anglaises, et Louise Tilly ${ }^{8}$, désireuse d'enraciner les troubles frumentaires dans la tradition, ont montré comme après eux, Denis Beliveau', que les acteurs de ces mouvements étaient hantés par le schème de "l'économie morale" et par le désir du recours au souverain. Ces historiens s'accordent à souligner combien les trublions veillaient à modeler leurs actes sur leurs propres représentations de la légalité et tenaient à la caution des autorités locales. Yvon Bionnier ${ }^{10}$, pour sa part, a révélé l'importance du territorial dans le déroulement de ces actes violents. Les "bandes" qui agitent les campagnes de l'Indre, au lendemain de l'émeute de Buzançais, sont construites sur une base communale. Ce jeune historien montre aussi comment, au cours de ce carnaval tragique, s'opère le transfert de la haine, qui ne vise plus tant le noble que l'usurier petit-bourgeois, héritier de "l'accapareur". Les violences antifiscales qui agitent les campagnes en 1814 et 1815, en 1830, en 1848 et 1849 sont, elles aussi, traditionnellement interprétées comme des archaïsmes. Les ruraux s'en prennent aux droits réunis, puis aux quarante-cinq centimes, en des temps qu'ils perçoivent être sous le signe de la transition du pouvoir. Ils nourrissent, en ces occurrences, l'espoir de la remise de l'impôt tandis que, plus confusément, ils refusent que soient prélevées sur le monde rural les sommes destinées au décollage de l'économie. A ce propos, la grande émeute de Gourdon, après celle d'Ajain, mériterait une analyse systématique. Les violences suscitées par le refus des normes nouvelles imposées par l'Etat, en matière de chasse, d'usage de la forêt ou de recrutement du contingent sont, elles aussi, interprétées, le plus souvent, comme autant de manifestations archaïques. Maurice Agulhon ${ }^{11}$, qui souligne le rôle des bois dans les différends qui opposent les communes du Var, John Merriman ${ }^{12}$, historien de la "guerre des Demoiselles" et des incendies normands, n'ont pas véritablement refusé cette interprétation, bien qu'on puisse discerner dans leurs travaux cette évolution des curiosités qui constitue notre propos. A dire vrai, durant près d'un demi-siècle, les historiens de la violence collective, particulièrement ceux qui ont travaillé sous la direction d'Ernest Labrousse, tentaient de discerner, derrière la réitération des gestes anciens, la manifestation d'une conscience de classe, le passage d'une mentalité pré-révolutionnaire à une mentalité révolutionnaire. Tout ce qui n'en était pas la marque ne pouvait, à leurs yeux, que désigner l'archaïsme. En bref, au coeur des années 1970, l'histoire de la violence rurale du premier XIXe siècle semblait en bonne voie; mais elle se construisait sous le signe d'une attente: celle d'une modernité politique. On guettait la pénétration des mouvements par les idéologies diffusées à partir des grands centres urbains. Les historiens posaient sur le monde des campagnes un regard vertical. Cette lecture, hantée par l'image de la propagation, dessinée selon le schème de la coulée, qui s'effectue de haut en bas d'une société pensée comme pyramidale, était, somme toute, peu attentive aux acteurs. La paysannerie française, dans cette perspective, est décrite comme perpétuellement en attente d'un message émis par la société englobante, comme une cire molle offerte aux apprentissages. Il semble qu'il n'y ait eu, pour elle, d'autre histoire possible que celle de l'obéissance, de la soumission à la médiation de notables ou celle de l'émancipation apportée de l'extérieur; le reste ne pouvant être que flambées de violence aveugle, que vestiges d'un autre âge. L'historien postulait 
implicitement la subordination des désirs des paysans à des idéaux transmis par des missionnaires,"rouges" le plus souvent - en effet, l'étude du césarisme démocratique a été, pour l'essentiel, laissée à des spécialistes du "bonapartisme". Il s'efforçait surtout de percevoir comment la violence archaïque était, à l'occasion, pénétrée, assumée, instrumentalisée par des militants républicains ou démocrates-socialistes. Si l'on respecte la chronologie des travaux, Philippe Vigier ${ }^{13}$ (région alpine), Maurice Agulhon ${ }^{14}$ (département du Var), Alain Corbin ${ }^{15}$ (Limousin), Pierre Lévêque ${ }^{16}$ (Bourgogne), Raymond Huard ${ }^{17}$ (département du Gard), Marcel Vigreux ${ }^{18}$ (Morvan) ont, tour à tour, adopté cette lecture de la violence rurale du premier XIXe siècle. Parallèlement, John Merriman ${ }^{19}$ et, d'une manière plus précise, Edward Berenson ${ }^{20}$, puis, Peter Mc Phee ${ }^{21}$ ont consacré des ouvrages de synthèse au processus de diffusion en nappe de l'idéologie républicaine et démocrate-socialiste dans les campagnes "rouges" du Centre, du Centre-Ouest et du Sud-Est. L'essentiel, dans cette perspective, était d'étudier la structure des réseaux de militants l'implantation des sociétés secrètes, l'action des colporteurs, des agents d'assurances, des voituriers, des migrants, des propagandistes envoyés par les organisations centrales, les procédures de diffusion des brochures, des catéchismes, des images, des statuettes, avant que la répression ne vienne, pour quelque vingt ans, enrayer le processus. Il n'est pas question de contester ici cette lecture du passé politique des campagnes françaises. La diffusion en nappe des idéologies s'est effectivement opérée, en certaines régions tout au moins, selon des modalités et selon des itinéraires qu'il importait de percevoir et de décrire. Reste qu'en ce qui concerne les communautés rurales, le risque est grand, pour qui adopte cette perspective, de confondre conversion idéologique et simple calcul opportuniste ou, si l'on préfère, instrumentalisation des procédures du débat national en vue de réaliser des objectifs définis localement. A tel point qu'on peut se demander si le temps n'est pas venu d'élaborer une histoire plus attentive à la réception. On sait combien fructueuse s'est révélée une telle démarche dans le domaine de l'histoire littéraire, de l'histoire des arts ou de l'histoire culturelle. On sent monter chez les spécialistes le besoin d'adopter une optique compréhensive, de se mettre en quête des logiques de comportement, des rationalités propres à la paysannerie. Abandonner le regard vertical implique un long travail préalable. On a trop vite postulé, en effet, dans la France du XIXe siècle, l'intégration à l'économie de marché, le caractère interpersonnel des relations, l'individualisme des pratiques, que ce soit dans le domaine des transactions, des comportements délictueux ou des attitudes électorales; on a trop vite décrété l'intégration à l'Etat-nation et l'adoption d'un système uniforme de valeurs et de normes sur l'ensemble du territoire. A ce propos, les historiens se sont souvent laissés piéger par les découpes opérées par les initiateurs, les réalisateurs ou les commentateurs des enquêtes du XIXe siècle et par les représentations sociales qui guidaient la plume des administrateurs. Simplification abusive qui, du moins, facilitait la perception d'éventuels reclassements, de solidarités nouvelles et donc des signes d'une conscience de classe. L'étude des territoires communautaires, des modalités de leur saisie, des procédures de leur marquage et des moyens de leur défense constitue la première des tâches préalables que je viens d'évoquer. Par bonheur, de Maurice Halbwachs et Marcel Maget à Marcel Roncayolo, anthropologues et géographes ont beaucoup fait progresser cette branche de l'histoire de la construction des territoires et de l'imaginaire de l'espace. Plusieurs historiens, notamment Marie-Noelle Bourguet, ont d'autre part aidé à déjouer le piège tendu,en ce domaine, par la pesée des schèmes néo-hippocratiques sur le regard des observateurs sociaux du premier XIXe siècle. 
L'histoire des comportements politiques présuppose, d'autre part, la connaissance des structures de la famille et de la parenté, réelle, fictive ou symbolique ainsi que celle des réseaux d'amicalité, de fidélité, de clientèle. En fonction de ces préalables, peut être analysée la configuration des sociétés d'interconnaissance qui constituent la communauté de hameau, la communauté villageoise ou, selon les régions, ces entités plus vastes que sont le "pays" ou la communauté de vallée, en région de montagne ${ }^{22}$. Sans, notamment, le repérage de l'aire de reconnaissance à l'intérieur de laquelle la défense de la réputation se révèle impérative, il est peu d'espoir de bien comprendre les comportements individuels et collectifs. L'histoire des temps sociaux constitue, à mon sens, le second travail indispensable à la compréhension de ce qui noue la violence au politique. On mesure mieux, désormais, le poids de la mémoire collective, le rôle de la gestion du souvenir des antagonismes, des violences, des événements qui ont marqué la communauté, affectée par tout ce qui a perturbé la société englobante. L'histoire des attitudes politiques dans les campagnes françaises du premier XIXe siècle est totalement incompréhensible à qui ignore la façon dont les souvenirs des révolutions et des guerres, voire ceux de l'Ancien Régime finissant, ont été construits, puis réaménagés ; ainsi que la manière dont ils ont dessiné les tensions, suscité d'éventuels cycles vindicatoires (Colin Lucas ${ }^{23}$, Raymond Verdier), ordonné la scénographie ultérieure de la violence collective. Tout aussi nécessaire, mais bien plus difficile, est de tenter de discerner comment s'élaborent et opèrent les représentations de l'avenir des groupes et des communautés ${ }^{24}$. Ce travail préalable permet d'étudier, tout à la fois, la conscience d'appartenance, la façon dont se construisent et s'entrelacent les identités, la prégnance des marqueurs symboliques. De telles analyses conduisent logiquement à celle de l'échelle des positions à l'intérieur de la communauté. Il importe, en effet, de saisir toutes ces hiérarchies fines, dessinées selon de multiples critères, qui ordonnent la gamme des conduites visant à la défense de l'honneur, du prestige ou de la simple réputation. Le plus difficile, à ce propos, est de saisir toutes ces données dans leur dynamisme même; de discerner la manière dont les identités, comme les échelles hiérarchiques, sont, en permanence, réaménagées ${ }^{25}$. Reste alors à écrire les pages les plus obscures de l'histoire politique des campagnes françaises : celles qui traitent des représentations du hors-groupe, du souverain, des centres de pouvoir, notamment de Paris, devenu capitale, et de la médiation attendue du notable. C'est en fonction de toutes ces données que s'effectuent les calculs auxquels se livrent en permanence les membres des diverses communautés. Leur action vise au maintien, et si possible à l'accroissement, ou au réaménagement des patrimoines, des usages, des systèmes de normes ${ }^{26}$ ainsi qu'à l'acquisition de "sécurités émotives invisibles". Le comportement politique résulte ici d'un jeu de désirs et d'attentes; et aussi de la nécessité de comprendre l'événement brutal ou le processus insidieux qui risquent de rompre les équilibres. Les communautés rurales doivent, en permanence, savoir comment interpréter et conjurer l'irruption d'une éventuelle menace ; il leur faut, bien souvent, adapter, pour cela, leur système de représentations du politique à la situation, opérer une nécessaire réduction des systèmes de causalité, trouver au besoin des victimes substitutives. Autant dire que l'étude de la rumeur s'impose à l'historien qui ne doit pas se laisser rebuter par son apparente incohérence. Tenter de percevoir la rationalité politique de la violence rurale implique donc une écoute attentive, impose de distinguer ce qui est refus $^{27}$ de ce qui est volonté d'intégration, d'adaptation ou d'anticipation. Alors seulement peut être entreprise l'étude de la stratégie ou, si l'on préfère, de l'ensemble des tactiques employées en vue de réaliser ces objectifs. L'usage 
de la violence s'intègre en effet à un ensemble de conduites qui trouve sa cohérence dans les desseins profonds de la communauté. Celle-ci vise, répétons-le, à la sauvegarde des équilibres. La vantardise, la dérision, notamment à l'égard du notable ou du "horsain", la menace, l'injure et le défi qui servent à déprécier, voire à affaiblir le groupe antagoniste ${ }^{28}$ constituent les plus élémentaires de ces tactiques. Au sein de la société rurale de ce temps, familles et individus sont perpétuellement en compte ${ }^{29}$. L'équilibre est assuré par un jeu complexe d'échanges, de compensations et, surtout, par la pratique de "l'arrangement" ${ }^{130}$. La dénonciation, la plainte et, d'une manière générale, le recours au judiciaire entrent dans la gamme des tactiques ${ }^{31}$, ainsi que, bien entendu, l'usage de la violence. On connait la fonction structurante de cette dernière ${ }^{32}$; elle est particulièrement évidente dans les campagnes du premier XIXe siècle. En ce milieu, la violence contribue clairement à l'approfondissement de la conscience d'appartenance et, parfois, au soulagement de l'angoisse qui tenaille une communauté assaillie par l'incompréhensible ${ }^{33}$. Son souvenir entre dans "l'héritage immatériel". Sans la connaissance des mécanismes de la violence qui se déchaîne entre les individus, les familles, les hameaux, les communes et leurs jeunesses ${ }^{34}$, entre les membres d'un même "pays" ou d'une même région ${ }^{35}$, il est peu de chances de comprendre le déroulement des troubles et des émeutes de portée plus large. La violence collective qui affleure à la surface de l'histoire nationale a pour trame celle qui est ancrée au coeur des communautés. Nous arrivons, enfin, aux interrogations posées initialement. Comment se nouent, entre 1814 et la fin des années 1850, la rationalité de la violence rurale et le déroulement du débat politique au village? Quels facteurs peuvent expliquer la retombée de l'agitation durant le dernier tiers du siècle? Afin de répondre à ces questions, il convient de souligner, tout d'abord, un processus trop souvent négligé. Dans les campagnes françaises, cette période se caractérise par l'ascension du rôle des autorités locales. Le pouvoir municipal constitue, en premier lieu, une instance décisive dans la régulation interne des conflits : le maire joue alors un rôle déterminant dans la procédure de "l'arrangement". Entouré du conseil municipal, il gère, d'autre part, la réponse qu'il convient d'apporter à l'injonction ou à la sollicitation extérieures ; c'est lui qui, bien souvent, décide de la résistance, du refus ou de l'acceptation. Cette ascension du municipal est particulièrement nette au cours des années 1830. La constitution, ou la reconstitution, d'une garde nationale dans la plupart des communes rurales au lendemain des événements de juillet, l'élection des officiers de cette garde, la célébration festive des libertés restaurées ont entamé le processus. La loi de mars 1831, qui a rendu électif le conseil municipal - sans oublier celle qui a conféré aux communes la qualité de personne civile - a bouleversé la politique au village. André-J. Tudesq, Philippe Vigier ${ }^{36}$, Rachel Gauducheau ont montré que cette mesure a conféré la qualité d'électeurs à presque tous les chefs de famille établis dans les communes rurales. Alors débute véritablement l'apprentissage du sufffrage. La commmunauté villageoise dispose dès lors d'une porte-parole autorisé. Le débat politique s'insinue, puis se déploie à l'occasion d'une gamme de micro-conflits - entre l'autorité écclésiastique et le pouvoir municipal notamment - que Judith Silver et Barnett Singer se sont efforcés d'analyser ${ }^{37}$. Désormais, la municipalité peut effectivement gérer la commune, sous le contrôle il est vrai, de l'autorité préfectorale. Cette gestion s'effectue en fonction de représentations mouvantes de la modernité, qui conduisent à focaliser successivement l'attention sur les objets suivants: reconstruction, agrandissement de l'église et du presbytère, reconstitution du trésor et de la sonnerie, déplacement du cimetière, amélioration ou création de chemins (1836), construction d'une place, choix d'un garde 
champêtre, installation d'une école, d'un bureau de poste, aménagement de fontaines et de lavoirs, instauration de foires ou de marchés, création d'une compagnie de pompiers, édification d'une mairie, "municipalisation" du territoire par la construction de monuments et par la multiplication d'objets symboliques. Parallèlement à cette action édilitaire, nombre de communes se préoccupent de l'enregistrement ou de la codification de ces innombrables usages locaux qui compensent l'absence de code rural (Louis Assier-Andrieu). C'est chez le maire - c'est-à-dire "à la mairie" - et au sein du conseil municipal que s'effectue le calcul des recours à la médiation. Alain Guillemin a naguère montré, à propos des notables de la Manche, combien l'emprise de ceux-ci était, en fait, soumise à de telles procédures. Christian Thibon ${ }^{38}$, en ce qui concerne le pays de Sault, Jean-François Soulet ${ }^{39}$, à propos de l'ensemble de la chaîne pyrénéenne, ont magnifiquement montré la logique de ces comportements collectifs. Le recours aux autorités municipales et l'intervention de celles-ci à l'occasion des troubles frumentaires, des luttes forestières ou des émeutes antifiscales s'intègrent logiquement à un tel processus. Le conseil municipal est aussi un porte-parole de la communauté quand il s'agit d'intervenir dans le débat politique national. On a trop négligé, à ce propos, la pratique de l'adresse, du voeu et de la pétition. On a considéré trop distraitement les registres de délibérations. Là peut parfois se lire, notamment, le sens de l'élection du 10 décembre 1848 ou celui de l'attachement à Napoléon III. C'est au maire enfin que revient, par ses allocutions prononcées au sortir de la messe, ce que dans certaines régions de l'Ouest on appelle alors le "prône de la mairie", une tâche pédagogique : à lui d'expliquer les libertés, au lendemain de juillet 1830, ou de lire et de commenter publiquement la constitution, en novembre 1848. Le ralliement à la république, cette année-là comme au cours des années 1870 (là où il s'est opéré), le soutien apporté à Louis-Napoléon Bonaparte puis à l'empereur, l'adhésion des ruraux de certaines régions au programme des démocrates-socialistes en 1849 doivent, dans la majorité des cas, être lus comme le résultat d'un calcul des communautés. C'est la seule façon d'expliquer l'apparente contradiction repérée depuis longtemps par les historiens entre les résultats de plusieurs scrutins successifs. Qu'en est-il, dans cette perspective - celle qui consiste à s'efforcer d'arrimer l'histoire politique à la lecture des documents qui émanent des communautés rurales - du basculement qui, à partir du milieu du siècle, se serait opéré selon le schéma suivant: force du territorial/du communautaire/du coutumier/intensité de la violence collective vers le triomphe du national/de la citoyenneté/application de la loi/pacification du territoire? Notons, tout d'abord, que le sentiment national, saisi à ce niveau, peut se lire, en 1814 et 1815, dans les réactions suscitées par l'occupation du territoire et qu'il s'impose, plus nettement encore, à l'observateur en 1870, avant même la chute du Second Empire (Stéphane Audouin-Rouzeau). Il convient donc de se garder de surestimer, à ce propos, l'ampleur de l'évolution accomplie sous la IIIe République. Cela dit, il ne saurait être question de nier le progrès de l'intégration à l'ensemble national (Eugen Weber) ${ }^{40}$, d'autant que ce processus contribue amplement à expliquer l'affaissement de la violence rurale. L'édification du réseau ferroviaire, la pratique ininterrompue du suffrage universel à partir de décembre 1851 (Patrick Lagoueyte), l'instauration de la conscription, la création de nouveaux moyens de maintien de l'ordre - Suzanne Coquerelle, Jean-Marc Berlière ${ }^{41}$ - et l'intolérance nouvelle à l'égard de la violence collective incontrôlée (Louise, Charles et Richard Tilly) ont, à l'évidence, contribué à cet apaisement. Mais il convient de se garder de lire cette pacification comme le signe d'une dissolution du territorial et du communautaire. On a beaucoup souligné cette 
intégration à l'Etat-nation, à son système de normes ainsi que la prise de conscience collective de son territoire, sans toujours dire suffisamment, me semble-t-il, qu'il s'agit d'une captation plus que d'une destruction. Le Second Empire et, mieux encore, la IIIe République (Christian Thibon) ${ }^{42}$ ont su, en effet, capter ce qui fondait la solidité des références à la localité ainsi que la force du communautaire, assumées depuis un demisiècle par le municipal. On connaît le sens de l'instauration d'un Sénat ou celui des banquets des maires (Jocelyne George); on se rappelle les discours de Jules Méline consacrés au clocher et à la mairie de village, le poids de l'idéologie agrarienne ${ }^{43}$, puis régionaliste ${ }^{44}$ et la manière dont certains historiens de l'économie ont déploré la puissance d'un grand parti des campagnes, qui aurait freiné la croissance. Plus significatifs encore sont, à mon sens, le "recentrement sur le clocher" de la peinture officielle de paysage, dont parle Françoise Cachin ${ }^{45}$ et la structure de cet harmonieux Tableau de la France dessiné par Vidal de la Blache pour la très républicaine Histoire de la France contemporaine, dirigée et en grande partie écrite par Ernest Lavisse. Dans nombre de régions, le village ou, plutôt, la commune rurale connaît, alors, un nouvel âge d'or. Certes, elle a perdu, du fait d'un exode sélectif, cette complexité sociale qui était la sienne à l'aube des années 1860 ; mais elle est devenue, par cette simplification même, beaucoup plus cohérente. Sa vie festive se trouve enrichie par la prolifération des organisations et des associations qui entretiennent une sociabilité intense ${ }^{46}$. A trop déplorer la terre qui meurt, processus indiscutable en certains points du territoire, on oublie que dans bien des régions, en Normandie par exemple, la communauté rurale, apaisée par la disparition partielle des tensions sociales qui la déchiraient, devenue moins anxieuse devant l'apport extérieur, se révèle plus soudée que naguère. Guidée par des autorités municipales mieux enracinées, plus sûres d'elles et qui n'hésitent plus à multiplier les arrêtés, la commune rurale demeure, à la fin du XIXe siècle, une cellule vivante, soumise plus que jamais au calcul de ses intérêts et aux représentations de son avenir. Si l'on compare l'historiographie récente à celle qui était dominante à l'aube des années 1970, on constate une évidente dérive. La violence rurale, en ses formes mouvantes et dans son mimétisme ordonné par les souvenirs des grands bouleversements, tend à être lue selon sa rationalité propre. A l'issue d'analyses minutieuses des communautés, elle apparaît de plus en plus étroitement liée à des désirs, à des attentes, à des représentations de l'avenir qui suggèrent des résistances à l'apport extérieur ou l'intégration de celui-ci selon de subtiles procédures de réaménagement. Dans cette perspective, la violence ne constitue qu'une arme, parmi d'autres, utilisée par des communautés qui tentent de s'adapter aux transformations du marché, aux conjonctures écologique et biologique ${ }^{47}$, aux systèmes de valeurs et de normes imposés de l'extérieur, aux nouvelles représentations de soi, de l'autre, du souverain ou du peuple citadin. Il convient désormais de s'efforcer de discerner, dans leur spécificité, ces processus de résistance et d'adaptation ; de relire la violence rurale, ses gestes, sa rhétorique avec d'autres lunettes que celles qui font des campagnes du premier XIXe siècle une simple terre de mission, tantôt secouée d'aveugles soubresauts, tantôt figée dans l'attente de l'idéologie salvatrice. 


\section{NOTES}

1. Bercé (Y-M.), Croquants et va-nu-pieds : les soulèvements paysans en France du XVIe au XIXe siècle. Paris, 1974.

2. Beliveau (Denis), thèse consacrée aux troubles frumentaires en France durant la première moitié du XIXe siècle, préparée sous la direction de Jacques Revel et soutenue en 1992 à l'EHESS.

3. Lefebvre (G.), Les Paysans du Nord pendant la Révolution française. Lille, Marquant, 1924. La grande peur de 1789. Paris, A. Colin, réed., 1988.

4. Pilbeam (P.), "Popular Violence in Provincial France after the 1830 Revolution", English Historical Journal, vol. 91, n 359, 1976."The Three Glorious Days, the Revolution of 1830 in Provincial France", Historical Journal, t. 26, n 4, 1983 ; Price (R.), "Popular Disturbances in the French Provinces after the July Revolution of 1830", European Studies Review, vol. 1, n4, 1971. The French Second Republic : A Social History. London, 1972. Corbin (A.), Archaisme et modernité en Limousin au XIXe siècle. Paris, Rivière, 1975. Le village des cannibales. Paris, Aubier, 1990. L'histoire de la violence dans les campagnes françaises au XIXe siècle. Esquisse d'un bilan". Ethnologie Française, XXI, 1991.3.

5. Soo Yun Chun, La propagande républicaine et la question paysanne au début de la Troisième République. Thèse, Univ. Paris I, 1990.

6. Op. cit.

7. Vigier (P.), La Seconde République dans la région alpine. Paris, P.U.F., 1963. La vie quotidienne en province et à Paris pendant les journées de 1848. Paris, Hachette, 1982. Gossez (R.), "La résistance à l'impôt : les quarante-cinq centimes", Etudes, 15, 1953. Bionnier (Y.), L'émeute de Buzançais, mémoire de maîtrise, Tours,1979.

8. Tilly (L.), "La révolte frumentaire, forme de conflit politique en France", Annales ESC, 27e année, $n^{\circ} 2,1972$.

9. Op. cit.

10. Bionnier (Y.), op. cit.

11. Agulhon (M.), La République au village. Paris, Plon, 1969.

12. Merriman (J.), "The Norman Fires of 1830 : Incendiaries and Fear in Rural France", French Historical Studies, 9. 1976. The Agony of the Republic: The Repression of the Left in Revolutionary France. 1848-1852. New Haven, (Connect.), 1978.

13. Vigier (P.), op. cit.

14. Agulhon (M.), op. cit.

15. Corbin (A.), op. cit.

16. Lévêque (P.), Une société provinciale : la Bourgogne sous la monarchie de juillet, Paris, EHESS, 1983.

17. Huard (R.), La préhistoire des partis : le parti républicain en Bas-Languedoc.

1848-1881. Paris, Fond. Nat. Sc. Pol., 1982.

18. Vigreux (M.), Paysans et Notables du Morvan au XIXe siècle. Château-Chinon, 1987.

19. Merriman (J.), op. cit.

20. Berenson (E.), Populist Religion and Left-Wing Politics in France,1830-1852.

Princeton, 1984.

21. Mc Phee (P.), The Politics of Rural Life. Political Mobilization in the French Countryside. 1846-1852. Oxford, Clarendon Press, 1992. 
22. Chiva (I.) et Goy (J.), Les Baronnies des Pyrénées, Genève, Droz, 1981. Soulet (J.F.), Les Pyrénées au XIXe siècle. Toulouse, Eché, 1987.

23. Lucas (C.), "Themes in Southern Violence after 9 Thermidor", in Beyond the Terror, Essays in French Regional and Social History, Cambridge, U.P., 1988.

24. Levi (G.), Le pouvoir au village : Histoire d'un exorciste dans le Piémont du XVIIe siècle, (Présenté par Jacques Revel), Paris, Gallimard, 1989.

25. Jessenne (J.P.), Pouvoir au village et révolution : Artois. 1760-1848. Lille, 1987.

26. Soulet (J.F.), op. cit.

27. Soulet (J.F.), op. cit. Maire (Catherine-Laurence), Les possédées de Morzine. 1857-1873. Lyon, P.U.L., 1981.

28. Claverie (E.) et Lamaison (P.), L'impossible mariage. Violence et Parenté en Gévaudan. XVIIe-XIXe siècle, Paris. Hachette. 1982.

29. Chauvaud (F.), Tensions et conflits. Aspects de la vie rurale au XIXe siècle d'après les archives judiciaires. L'exemple de l'arrondissement de Rambouillet (1811-1871), thèse, université Paris X, janvier 1989.

30. Ploux (F.), op. cit.

31. Claverie (E.) et Lamaison (P.), op. cit. Poucher (Y.), Les maîtres de granit. Paris, Orban, 1987. Grandcoing (P.), "La bande à Burgout". Limoges, Souny, 1991.

32. Coser (L.), Les fonctions du conflit social. Paris, P.U.F., 1982. Maffesoli (Michel), Essais sur la violence, banale et fondatrice. Paris, Lib. des Méridiens, 1984.

33. Corbin (A.), op. cit.

34. Ploux (F.), "Les bagarres de village (1815-1850)", Ethnologie française, XXI, 1991. 3.

35. Cf. l'attitude des migrants temporaires installés à Paris.

36. Op. cit.

37. Silver (J.), "French Peasant Demands for Popular Leadership in the Vendômois (Loir-et-Cher), 1852-1890", Journal of Social History, 14.1980. Singer (B.), Village Notables in 19th Century France. Albany, 1983.

38. Thibon (C.), Pays de Sault, les Pyrénées audoises au XIXe siècle : les villages et l'Etat, Paris, éd. du CNRS, 1988.

39. Op. cit.

40. Weber (E.), Peasants into Frenchmen. Stanford, U.P., 1976.

41. Berlière (J-M.), thèse consacrée à l'histoire de la police en France durant la seconde moitié du XIXe siècle, préparée sous la direction de Pierre Lévêque et soutenue en janvier 1991 à l'université de Dijon.

42. Thibon (C.), op. cit.

43. Barral (P.), Les agrariens français de Méline à Pisani, Paris, A. Colin, 1963.

44. Thiesse (A-M.), Ecrire la France, Paris, P.U.F., 1988. Brustein (W.), Social Origins of Political Regionalism. France 1849-1981, Berkeley, 1988.

45. In Les Lieux de Mémoire. sous la direction de Pierre Nora.

46. Agulhon (M.) et Bodiguel (M.), Les associations au village. Le Paradou, Actes-Sud, 1981.

47. Op. cit. 


\section{RÉSUMÉS}

Longtemps l'histoire de la violence rurale dans la France du XIXè siècle s'est construite sous le signe d'une attente : celle d'une modernité politique. Les historiens guettaient la pénétration des mouvements par les idéologies diffusées à partir des grands centres urbains. Cette lecture de la violence hantée par l'image de la coulée qui s'effectue de haut en bas d'une société pensée comme pyramidale, était peu attentive aux acteurs. La paysannerie était présentée comme une cire molle, en attente d'un message émis par la société englobante. Elle paraissait n'avoir eu d'autres choix possibles que la soumission à la médiation des notables ou le consentement à une émancipation apportée de l'extérieur. Le temps est venu d'élaborer une histoire plus attentive à la réception. Il convient désormais de se mettre en quête des logiques de comportement, des rationalités propres à la paysannerie, tout en évitant de se laisser piéger par la séduction du tableau décrété par les observateurs sociaux du XIXè siècle. Cette relecture de la violence rurale doit notamment tenir compte des temps sociaux, des modalités du réaménagement permanent des souvenirs, de la manière dont se construisent et s'entrelacent les identités, dont s'effectue le calcul de l'avenir. Pour qui vise à comprendre le jeu de désirs et d'attentes ainsi que le système de représentations qui ordonnent le comportement politique, la scène municipale constitue un observatoire privilégié.

\section{INDEX}

Index géographique : France

Index chronologique : 19e siècle

Mots-clés : histoire, violence, ruralité 